\title{
Body mass index may be associated with outcomes in patients successfully resuscitated from cardiogenic arrest: a multi-center study
}

\author{
Chih-Wei Sung ${ }^{1}$; Joyce Tay ${ }^{2}$; Meng-Che Wu ${ }^{1}$; Jia-How Chang ${ }^{1}$; Chien-Hua Huang ${ }^{2}$; Wen-Jone Chen ${ }^{2}$; Wei-Tien Chang ${ }^{2}$; Min-Shan Tsai ${ }^{1,2}$
}

${ }^{1}$ Department of Emergency Medicine, National Taiwan University Hospital Hsin-Chu Branch, Hsinchu, Taiwan ; ${ }^{2}$ Department of Emergency Medicine, National Taiwan University Medical College and Hospital, Taipei, Taiwan

\section{BACKGROUND \& AIM}

The role of body mass index (BMI) on clinical outcomes in patients resuscitated from cardiac arrest has drawn attention recently. However, influence of obesity on clinical outcomes of patients underwent cardiac arrest is still controversial. This study is to investigate the association between $\mathrm{BMI}$ and outcomes in cardiac survivors.

\section{METHODS}

The retrospective cohort study recruited 273 non-traumatic adult cardiac arrest survivors who received emergent coronary angiography (CAG) after return of spontaneous circulation (ROSC) in three hospitals from January 2011 to September 2017. The enrolled patients were divided into the underweight, normal, overweight and obese groups based on BMI (underweight: $\mathrm{BMI}<18.5$; normal: 18.5-24.9; overweight: $25.0-29.9$; obese $\geq 30$ ). The neurological recovery was assessed by cerebral performance scale (CPC). The inhospital mortality and poor neurological outcome were compared between different BMI groups.

\section{RESULTS}

There were $13(4.8 \%)$ patients in the under-weight group, $121(44.3 \%)$ patients in the normalweight group, $100(36.6 \%)$ patients in the over-weight group and $39(14.3 \%)$ patients in the obese group. The obese group had significantly higher rates of inhospital mortality and poor neurological outcome $(\mathrm{CPC}=3 \sim 5)$ as compared to the other 3 groups (inhospital mortality: underweight $38.5 \%$, normal: $29.8 \%$, overweight: $39.0 \%$, obese: $64.1 \%, p=0.002$; poor neurological outcome: underweight $53.9 \%$, normal: $43.8 \%$, over-weight: $47.0 \%$, obese: $71.8 \%, p=0.02$ ). Compared with the normal weight group, the obese group presented with higher risk for inhospital mortality and poor neurological outcome (inhospital mortality: adjusted $\mathrm{HR}=5.24,95 \% \mathrm{Cl} 2.30-11.92, p<0.001$; poor neurological outcome: adjusted $\mathrm{HR}=3.84$, 95\% $\mathrm{Cl} 1.68-8.78, p=0.001$ ).

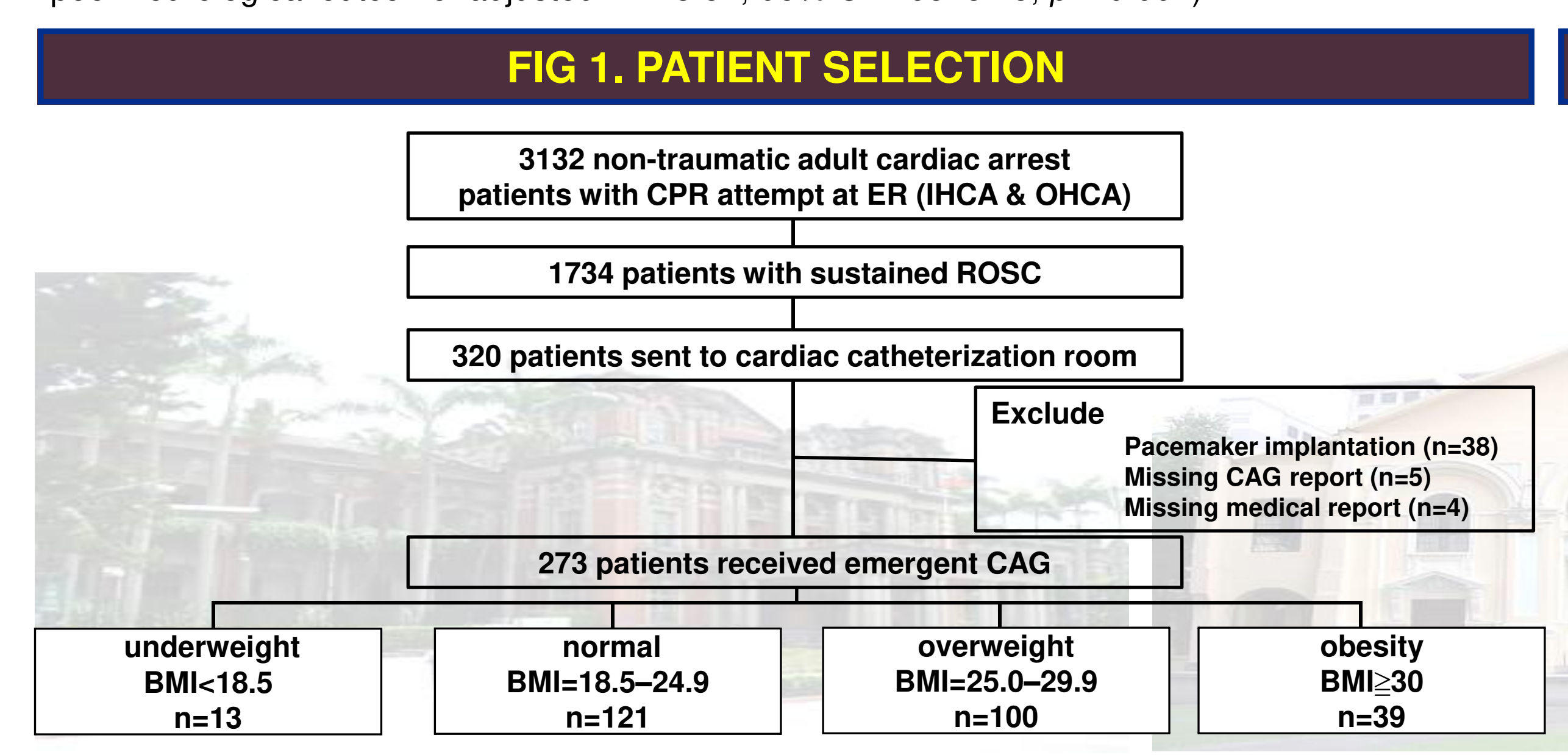

\section{TABLE 1. BASELINE CHARACTERISTICS}

\begin{tabular}{lcccccc}
\hline & \multicolumn{7}{c}{ Body mass index } \\
\cline { 2 - 6 } & total patients & underweight $<18.5$ & normal 18.5-24.9 & overweight 25.0-29.9 & obesity $\geqq 30$ \\
\cline { 2 - 6 } n (\%) & 273 & $13(4.76 \%)$ & $121(44.32 \%)$ & $100(36.63 \%)$ & $39(14.29 \%)$ & $p$ \\
\hline Age (years) & $60.84 \pm 13.27$ & $65.74 \pm 16.31$ & $61.00 \pm 13.75$ & $61.33 \pm 12.08$ & $57.46 \pm 13.39$ & 0.217 \\
Gender (Male) & $208(76.19 \%)$ & $10(76.92 \%)$ & $89(73.55 \%)$ & $77(77.00 \%)$ & $32(82.05 \%)$ & 0.743 \\
Smoking & $68(24.91 \%)$ & $3(23.08 \%)$ & $29(23.97 \%)$ & $25(25.00 \%)$ & $11(28.21 \%)$ & 0.959 \\
Hypertension & $154(56.41 \%)$ & $6(46.15 \%)$ & $60(49.59 \%)$ & $63(63.00 \%)$ & $25(64.10 \%)$ & 0.136 \\
Diabetes mellitus & $108(39.56 \%)$ & $1(7.69 \%)$ & $46(38.02 \%)$ & $42(42.00 \%)$ & $19(48.72 \%)$ & 0.044 \\
Dyslipidemia & $57(20.88 \%)$ & $3(23.08 \%)$ & $30(24.79 \%)$ & $18(18.00 \%)$ & $6(15.38 \%)$ & 0.498 \\
Coronary artery disease & $97(35.53 \%)$ & $4(30.77 \%)$ & $39(32.23 \%)$ & $37(37.00 \%)$ & $17(43.59 \%)$ & 0.593 \\
Chronic kidney disease & $49(17.95 \%)$ & $0(0.00 \%)$ & $21(17.36 \%)$ & $20(20.00 \%)$ & $8(20.51 \%)$ & 0.343 \\
Witnessed collapse & $222(81.92 \%)$ & $11(84.62 \%)$ & $103(85.83 \%)$ & $81(81.00 \%)$ & $27(69.23 \%)$ & 0.136 \\
Bystander CPR & $199(73.43 \%)$ & $8(61.54 \%)$ & $89(74.17 \%)$ & $76(76.00 \%)$ & $26(66.67 \%)$ & 0.483 \\
Initial shockable rhythm & $114(41.76 \%)$ & $4(30.77 \%)$ & $54(44.63 \%)$ & $43(43.00 \%)$ & $13(33.33 \%)$ & 0.489 \\
Shockable rhythm ever & $169(62.13 \%)$ & $9(69.23 \%)$ & $78(65.00 \%)$ & $60(60.00 \%)$ & $22(56.41 \%)$ & 0.697 \\
Heart rate at ROSC (BPM) & $101 \pm 26$ & $98 \pm 22$ & $102 \pm 25$ & $98 \pm 29)$ & $107 \pm 23$ & 0.289 \\
Inotrope use & $204(75.56 \%)$ & $8(61.54 \%)$ & $84(71.19 \%)$ & $75(75.00 \%)$ & $37(94.87 \%)$ & 0.012 \\
Therapeutic hypothermia & $98(39.84 \%)$ & $5(38.46 \%)$ & $51(46.36 \%)$ & $30(30.00 \%)$ & $12(35.29 \%)$ & 0.301 \\
Dual anti-platelet agents & $199(73.70 \%)$ & $8(61.54 \%)$ & $85(71.43 \%)$ & $76(76.00 \%)$ & $30(76.92 \%)$ & 0.573 \\
\hline
\end{tabular}

\section{TABLE 2. INHOSPITAL MORTALITY \& NEUROLOGICAL OUTCOME}

\begin{tabular}{|c|c|c|c|c|c|c|}
\hline & Inhospital mortality & Adjusted HR (95\% Cl) & $p$ & Poor neurological outcome & Adjusted HR (95\% Cl) & $p$ \\
\hline underweight $<18.5$ & $5(4.76 \%)$ & $1.17(0.35,3.97)$ & 0.799 & $7(5.19 \%)$ & $1.23(0.38,3.99)$ & 0.733 \\
\hline normal $18.5-24.9$ & $36(34.29 \%)$ & Reference & & $53(39.26 \%)$ & Reference & \\
\hline overweight 25.0-29.9 & $39(37.14 \%)$ & $1.55(1.01,2.76)$ & 0.043 & 47 (44.76\%) & $1.15(0.66,1.98)$ & 0.626 \\
\hline Obesity $\geqq 30$ & $25(23.81 \%)$ & $5.24(2.30,11.92)$ & $<0.001$ & $28(20.74 \%)$ & $3.84(1.68,8.78)$ & 0.001 \\
\hline
\end{tabular}

FIG 2. SURVIVAL CURVES IN BMI GROUPS

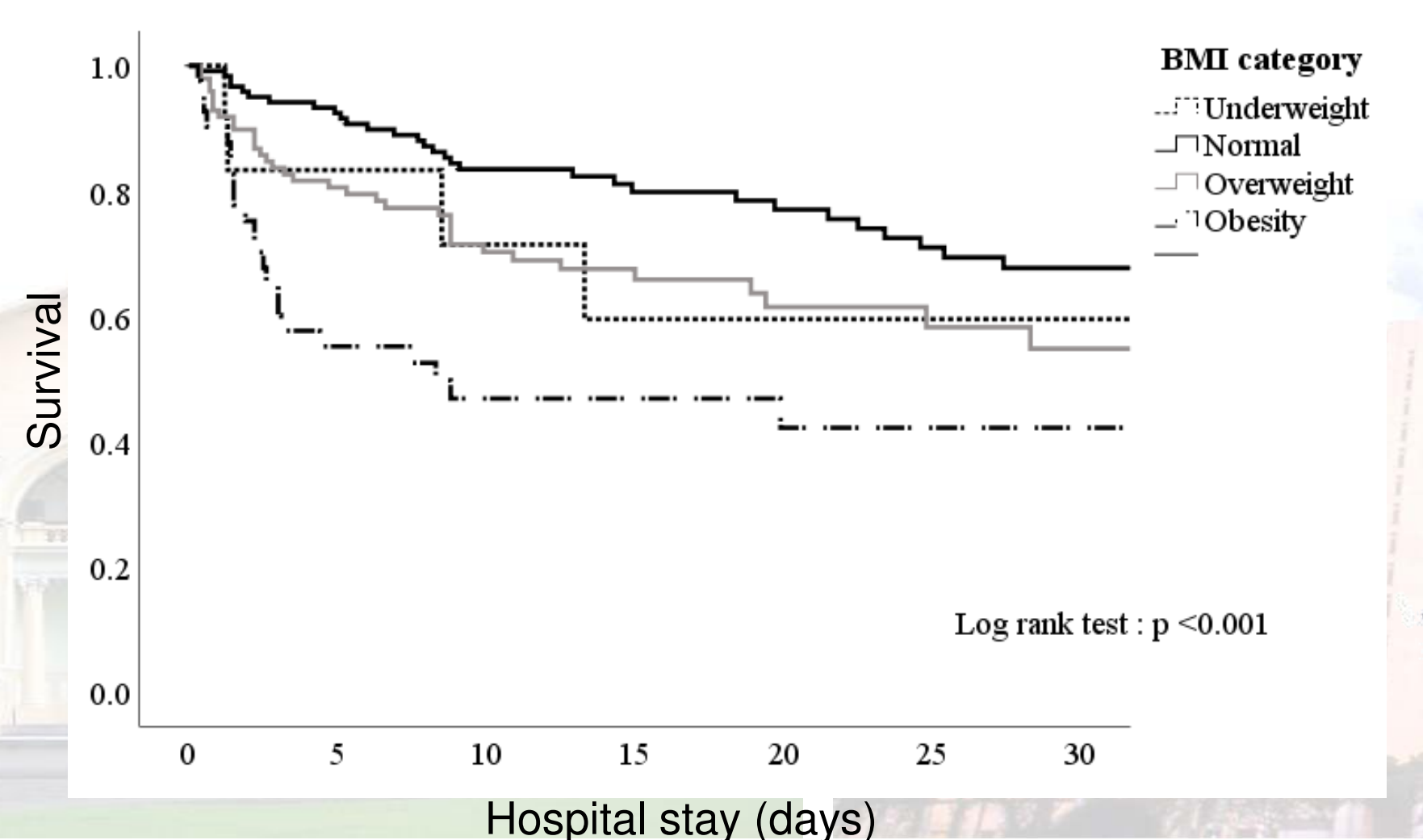
Hospital stay (days)

\section{CONCLUSIONS}

In patients with cardiogenic arrest, the obese BMI was associated with increased risk for in-hospital mortality and poor neurological recovery.

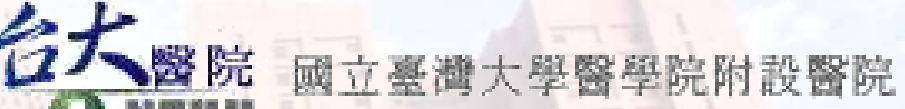

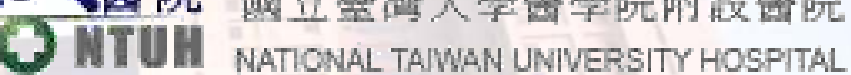
台大 